\title{
BK viremia and polyomavirus nephropathy in 352 kidney transplants; risk factors and potential role of mTOR inhibition
}

\author{
Johannes Jacobi ${ }^{1 *}$, Antonina Prignitz ${ }^{1}$, Maike Büttner ${ }^{2}$, Klaus Korn $^{3}$, Alexander Weidemann ${ }^{1}$, Karl F Hilgers ${ }^{1}$, \\ Katharina Heller ${ }^{1}$, Joachim Velden ${ }^{2}$, Antje Knöll ${ }^{3}$, Bernd Wullich ${ }^{4}$, Christoph May ${ }^{5}$, Kai-Uwe Eckardt ${ }^{1}$ \\ and Kerstin U Amann ${ }^{2}$
}

\begin{abstract}
Background: Polyomavirus BK nephropathy (PYVAN) remains an important cause of early graft dysfunction and graft loss in kidney transplantation.

Methods: In this retrospective, single centre cohort study we studied the incidence and outcome of BK viral infection in 352 patients transplanted in 2008-2011.

Results: During follow-up viral replication was detected in 48 patients (13.6\%); 22 patients (6.2\%) had biopsy proven PyVAN.

In multivariate logistic regression analyses risk factors for BK-viremia were lack of enrolment into randomized controlled trials (RCTs), biopsy proven acute rejections, cytomegaly virus (CMV) serostatus of both donor and recipient and previous transplantation.

In patients without PyVAN reduction or switch of immunosuppression was associated with rapid viral clearance and stable graft function. In contrast, in most patients with PyVAN graft function deteriorated and 5 patients prematurely lost their allograft. Switch of immunosuppression to a low dose cyclosporine plus mTOR inhibitor based regimen in patients with PyVAN was safe, well tolerated and tended to be associated with a better short-term outcome in terms of graft function compared to reduction of existing immunosuppression alone.

Conclusions: With the lack of licensed anti-polyoma viral drugs reduction or conversion of immunosuppression remains the mainstay of therapy in patients with PyVAN. The combination of low dose cyclosporine plus mTOR inhibition appears to be safe and warrants further investigation.
\end{abstract}

Keywords: Polyomavirus BK nephropathy, PyVAN, mTOR inhibition

\section{Background}

Recent advances in transplant immunology have led to improved allograft and patient survival following solid organ transplantation. Biopsy-proven acute rejection rates in kidney transplant recipients are now as low as $~ 10 \%[1,2]$. While short-term outcome following kidney transplantation is excellent, poor long-term allograft survival remains an unmet issue. One downside of more potent immunosuppressive drugs is the rise of opportunistic infections that

\footnotetext{
*Correspondence: johannes.jacobi@uk-erlangen.de

'Department of Nephrology and Hypertension, Friedrich-Alexander-University Erlangen-Nürnberg, Ulmenweg 18, 91054, Erlangen, Germany Full list of author information is available at the end of the article
}

may trigger premature graft failure. Of these, polyomavirus nephropathy (PyVAN) has caught special attention within recent years [3]. This virus, better known as BK virus belongs to the family of polyomaviridae, a group of small double-stranded DNA viruses [4].

Inapparent spread of infection occurs early in childhood and seroprevalence among the general population is high $(\sim 80 \%)[5,6]$. The virus has a specific tropism for the urogenital epithelium that represents a site of viral latency. BK virus associated pathology primarily occurs in immunocompromised patients. Among solid organ transplant recipients it is largely restricted to kidney transplantion. In this group of patients the prevalence of viruria, viremia

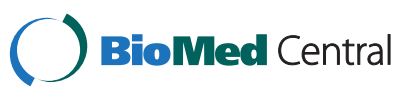


and PyVAN is as high as 30,13 , and $8 \%$, respectively [7]. It is still under debate whether reactivation of latent BK virus is host or donor-derived. Renal damage caused by BK virus comprises progressive tubulointerstitial nephritis and ureteral stenosis with a considerable risk of subsequent graft failure in $15-50 \%$ of cases $[8,9]$.

Known risk factors for the development of PyVAN are recipient as well as donor age, recipient race (white) and gender (male), HLA mismatches, previous biopsy proven acute rejections (BPAR), type of immunosuppression (i.e. tacrolimus and mycophenolate mofetil), use of antilymphocyte therapy and ureteral stent placement $[10,11]$.

To date, there is no effective antiviral therapy against PyVAN. The mainstay in the management of affected patients is the reduction or conversion of triple immunosuppression [12]. Other treatment options include the use of fluoroquinolones, intravenous immune globulines, leflunomide or cidofovir. The lack of specific targeted therapies has prompted a pre-emptive active surveillance strategy with routine screening intervals post transplantation for viral replication using PCR assays [13].

In the present study we retrospectively analyzed the incidence of BK viremia and PyVAN, the duration of viral replication and the short term outcome following different treatment strategies to achieve viral clearance.

\section{Methods}

\section{Study cohort}

In this retrospective single centre cohort study all patients $>18$ years who received a renal allograft at the University Clinic Erlangen during a four year period (2008-2011) were included. Patients were referred for transplantation from $\sim 40$ different non-profit or forprofit dialysis centres.

Ureteral stents were placed in all patients for the first 68 weeks after transplantation. Standard perioperative antibiotic regimen consisted of ampicillin/sulbactame for the first 10 days. CMV prophylaxis was administered according to current guidelines [13]. In all patients initial baseline triple immunosuppression included a calcineurininhibitor (CNI; either tacrolimus or CyA), antimetabolite (mycophenolate-sodium or mycophenolate mofetil) and steroids.

All patients gave their written informed consent for data collection and analysis prior to transplantation. All data were collected in strictly pseudonymous form.

Based on the retrospective nature of this cohort study and the fact, that patients were switched from one approved immunosuppressive regimen to another, this internal treatment guideline was not reviewed by our local ethics committee. However, all patients as well as outside treating physicians were informed about the purpose of reduction or conversion of immunosuppression.

\section{BK-screening and management of BK viremia and PyVAN}

In all patients screening for BK viremia was recommended at 3, 6, 9 and 12 months post transplantation. At months 3 and 12 blood samples were obtained while patients were undergoing recommended protocol biopsies, at the remaining time points samples were collected in our outpatient clinic. All transplant biopsies were stained for SV40 antigen and analyzed according to Banff criteria [14]. All patients with documented BK viremia underwent additional transplant biopsies at the time of diagnosis of viral replication to confirm or rule out the presence of PyVAN. In these patients follow-up biopsies were performed at the discretion of the treating physician. In patients with BK viremia viral load was measured every 6-8 weeks until at least two blood samples were negative for BK.

Viral replication was detected by real time PCR with sequences of probes and primers chosen from conserved regions of the $\mathrm{BK}$ virus (capsid and $\mathrm{T}$-antigen) genome as previously described [15]. The cut-off for this assay is 500 copies/ml.

In the presence of $\mathrm{BK}$ viremia the following treatment options are advocated in our transplant centre. In patients with low level viremia $\left(10^{3}-10^{4}\right.$ copies $\left./ \mathrm{ml}\right)$ without histological evidence for PyVAN, reduction of baseline immunosuppression (CNI 30\% and mycophenolate mofetil $50 \%$ ) is recommended. In cases of low immunological risk or further rise of viremia despite reduction of immunosuppression, these patients are switched to a low CyA $\left(\mathrm{C}_{0}\right.$ level: $60-80 \mathrm{ng} / \mathrm{ml}$ ) plus mTORi (trough level: $5-8 \mathrm{ng} / \mathrm{ml}$ ) based immunosuppressive regimen at the discretion of the treating physician. All patients with biopsy proven PyVAN and viral replication $>10^{4}$ copies $/ \mathrm{ml}$ are switched to a low CyA plus mTORi based regimen as described above, whenever feasible. In patients with high immunological risk (high levels of panel reactive antibodies, donor specific antibodies, antibody mediated or severe cellular rejection episodes prior to the onset of BK viremia) and in patients with eGFR $<20 \mathrm{ml} / \mathrm{min}$ and/or proteinuria $>1.0 \mathrm{~g} / \mathrm{g}$ creatinine reduction of current immunosuppression or switch of immunosuppression to a regimen other than low CyA plus mTORi is recommended.

\section{Statistical analysis}

Data (all biopsy results and relevant laboratory data within the first year) were collected and analysed using SPSS (Version 18.0). Continuous variables were summarized using descriptive statistics. Categorial variables were summarized using frequency tables and analyzed using ChiSquare test. Unpaired t-test or one-way ANOVA with posthoc Bonferroni adjustment was applied for subgroup analyses. Univariate and multivariate logistic regression analyses were performed to identify determinants and predictors for BK viremia in transplant recipients. Bar graph figures and results within the text are given as mean $\pm \mathrm{SD}$. 
Statistical significance was accepted at a value of $\mathrm{p}<0.05$ (2-sided).

\section{Results}

\section{Study cohort}

A total of 352 transplantations were included. Of these, $269(76 \%)$ were deceased donor transplants $(\mathrm{n}=198$ recipients $\leq 65$ years, $\mathrm{n}=71$ recipients $>65$ years) and 83 ( 24\%) living donor transplants $(\mathrm{n}=61 \mathrm{AB} 0$-compatible, $\mathrm{n}=22$ AB0-incompatible). In 22 patients simultaneous pancreas-kidney (SPK) transplantation was performed. In 9 recipients $>65$ years with expanded criteria donors transplantation of two kidneys was performed. Mean follow-up was $22.2 \pm 13.9$ months and did not differ between different subgroups treated for BK viral infection. Death censored one year allograft survival was $92.9 \%$, patient survival at one year was $96.6 \%$. Seven patients died with a functioning graft, another five patients died after having lost or without ever having graft function. Baseline characteristics and transplant relevant data of the entire study cohort, subgroups as well as patients with and without BK viremia are shown in Tables 1 and 2 .

\section{Transplant biopsies and BPAR within the first year after transplantation}

Within the first year 1218 transplant biopsies (including zero-hour biopsies) were performed. At 3 months 262 patients (74.4\%) underwent transplant biopsies, 67 biopsies were done for indication. The overall rate of BPAR at 3 months was $17.2 \%$ and significantly differed between patients with protocol biopsies (12.3\%) vs. biopsies done for indication $(31.3 \%, \mathrm{p}=0.001)$. At 12 months 188 patients $(53.4 \%)$ underwent transplant biopsies. The overall rate of BPAR at 12 months was $10.1 \%$ ( $n=11$ Banff IA, $\mathrm{n}=1$ Banff IIA, $\mathrm{n}=7$ subclinical humoral rejection episodes with detection of donor specific antibodies).

\section{Incidence, time course and risk factors for BK viremia and PyVAN}

During the study period BK viremia was detected in 48 patients $(13.6 \%$ of the entire cohort, Figure 1A). Of these, 36 patients were male ( $15.5 \%$ of all males) and 12 patients were female ( $10.0 \%$ of all females, $\mathrm{p}=\mathrm{n}$.s.). In 22 patients (6.2\% of the entire study cohort) renal biopsies confirmed the presence of PyVAN (Figure 1A). The frequency of BK viremia and PyVAN differed between subgroups, the highest incidence was observed in recipients of deceased donor allografts $>65$ years of age (Figure 1A).

Interestingly, all but one patient (preemptive transplant recipient) with $\mathrm{BK}$ viremia were on hemodialysis prior to transplantation (Table $1, \mathrm{p}=0.011$ ). The use of CNI, induction therapy and HLA-mismatch did not differ between patients with or without BK viral infection
(Table 2). However, patients with BK viremia were significantly older than patients without viral replication (Table 1) while donor age was similar (Table 2).

Onset of BK viremia was noted after $182 \pm 157$ days, or $\sim 6$ months after transplantation (see Additional file 1: Table S1 and Figure 1B). In patients with biopsy proven PyVAN the diagnosis of BK viremia was made later than in individuals without histological evidence for BK nephropathy ( $230 \pm 189$ vs. $141 \pm 113$ days, $\mathrm{p}=0.050)$. In 31 of the 48 patients with BK infection (64.6\%) onset of viremia occurred between days $60-180$ post transplantation (months 3-6), in 2 patients (4.2\%) viremia was present before the third month after transplantation, in 3 patients (6.2\%) with documented absence of viremia within the first twelve months BK viremia occurred between days $529-775$ post transplantation. The remaining 12 patients $(25.0 \%)$ did not undergo routine screening for viral replication as recommended (Figure 1B). In most of these individuals diagnosis was made while patients were admitted to undergo 1-year follow-up protocol biopsies.

On average, each patient underwent 2.8 PCR screenings for BK viral replication within the first year. Of all patients with at least one month graft survival 48 (14.4\%) had no blood screening for BK viremia within the first year. However, 33 of these patients had either lost their allograft by month $3(n=5)$ or had a protocol biopsy with absence of SV40 staining at this time point $(n=28)$, so that the diagnostic coverage was complete.

Initial viral load of patients with BK viremia was $109.587 \pm 245.821$ copies $/ \mathrm{ml}$. Based upon the various time points of detection of viral replication viral load increased with time after transplantation (Figure 1B, p= 0.11). Overall, there was a significant correlation between time of onset of viremia following transplantation and initial viral load $(\mathrm{r}=0.34, \mathrm{p}=0.019$, Figure 2A). In patients with biopsy proven PyVAN initial and peak viral loads were 1-log scale higher compared to patients with BK viremia without histological evidence for PyVAN, but significant overlap did not allow distinction. There was a strong correlation between initial and peak viral load $(\mathrm{r}=0.84, \mathrm{p}=0.0008)$, overall viral replication did not differ between different treatment groups of patients with either BK viremia or PyVAN (Figure 2B).

Using univariate logistic regression analyses the following variables were associated with BK viral replication (Table 3): ESP recipient status, recipient age, prior biopsy proven acute rejections (BPAR), lack of participation in prospective clinical transplant trials, mode of renal replacement therapy prior to transplantation, previous transplantation, donor (IgG -) as well as recipient $(\mathrm{IgG}+)$ CMV-serostatus, LDL-cholesterol, and - with borderline significance - 25-hydroxy vitamin D level. Other known risk factors such as baseline CNI (tacrolimus), induction therapy or later use of ATG, HLA 
Table 1 Baseline characteristics of transplant recipients

\begin{tabular}{|c|c|c|c|c|c|c|c|c|c|}
\hline Variable & $\begin{array}{l}\text { All patients } \\
(\mathrm{n}=352)\end{array}$ & $\begin{array}{c}\text { Deceased <65y } \\
(n=198)\end{array}$ & $\begin{array}{c}\text { Deceased >65y } \\
\quad(n=71)\end{array}$ & $\begin{array}{l}\text { LivingABO }_{c} \\
(n=61)\end{array}$ & $\begin{array}{l}\text { Living } \mathrm{ABO}_{\mathrm{i}} \\
\quad(\mathrm{n}=22)\end{array}$ & $\begin{array}{l}\text { ANOVA or } \\
\text { chi }^{2} p \text {-value }\end{array}$ & $\begin{array}{c}\text { No BK } \\
(n=304)\end{array}$ & $\begin{array}{c}\text { BK } \\
(n=48)\end{array}$ & $\begin{array}{c}\text { T-test or } \\
\text { chi }^{2} \text { p-value }\end{array}$ \\
\hline Age (years) & $51.4 \pm 13.5$ & $48.7 \pm 10.9$ & $67.7 \pm 2.7$ & $43.0 \pm 13.8$ & $45.4 \pm 11.5$ & 0.0003 & $50.5 \pm 13.4$ & $56.9 \pm 12.8$ & 0.002 \\
\hline Sex (male/female) & $232 / 120$ & $133 / 65$ & $51 / 20$ & $35 / 26$ & $13 / 9$ & n.s. & $196 / 108$ & $36 / 12$ & n.s. \\
\hline BMI $\left(\mathrm{kg} / \mathrm{m}^{2}\right)$ & $25.2 \pm 3.9$ & $24.8 \pm 4.0$ & $26.3 \pm 3.9$ & $24.9 \pm 3.7$ & $26.4 \pm 3.4$ & 0.013 & $25.2 \pm 4.1$ & $25.6 \pm 3.1$ & n.s. \\
\hline 1st, 2nd, 3rd, 4th transplant & $313 / 30 / 8 / 1$ & $172 / 20 / 5 / 1$ & $67 / 4 / 0 / 0$ & $54 / 5 / 2 / 0$ & $20 / 1 / 1 / 0$ & n.s. & $274 / 24 / 6 / 0$ & $39 / 6 / 2 / 1$ & 0.035 \\
\hline Blood group $(0, A, B, A B)$ & $130 / 157 / 39 / 26$ & $68 / 86 / 27 / 17$ & $25 / 36 / 7 / 3$ & $27 / 26 / 2 / 6$ & $10 / 9 / 3 / 0$ & n.s. & $108 / 137 / 37 / 22$ & $22 / 20 / 2 / 4$ & n.s. \\
\hline CMV IgG positive (n/\%) & $214 / 60.8$ & $123 / 62.1$ & 49 / 69.0 & $32 / 52.5$ & $10 / 45.5$ & n.s. & $178 / 58.6$ & $36 / 75.0$ & 0.020 \\
\hline Waiting time (months) & $40.8 \pm 36.4$ & $58.2 \pm 35.6$ & $25.6 \pm 25.9$ & $10.3 \pm 13.6$ & $18.0 \pm 24.9$ & 0.0007 & $41.4 \pm 36.8$ & $36.9 \pm 33.9$ & n.s. \\
\hline Dialysis vintage (months) & $54.2 \pm 40.3$ & $74.6 \pm 37.4$ & $39.1 \pm 24.0$ & $18.6 \pm 24.2$ & $18.5 \pm 26.3$ & 0.0003 & $54.8 \pm 40.1$ & $50.4 \pm 41.7$ & n.s. \\
\hline HD / PD / preemptive (n) & $292 / 40 / 20$ & $167 / 27 / 4$ & $67 / 4 / 0$ & $42 / 8 / 11$ & $16 / 1 / 5$ & 0.0001 & $245 / 40 / 19$ & $47 / 0 / 1$ & 0.011 \\
\hline Residual diuresis (ml/day) & $614 \pm 781$ & $370 \pm 616$ & $625 \pm 681$ & $1178 \pm 913$ & $1200 \pm 925$ & 0.0005 & $627 \pm 796$ & $526 \pm 680$ & n.s. \\
\hline Systolic BP (mmHg) & $140.1 \pm 18.7$ & $139.9 \pm 19.6$ & $143.9 \pm 20.0$ & $136.9 \pm 15.5$ & $138.7 \pm 13.4$ & n.s. & $140.5 \pm 18.8$ & $137.4 \pm 18.1$ & n.s. \\
\hline Diastolic BB (mmHg) & $81.4 \pm 10.8$ & $81.8 \pm 11.6$ & $80.5 \pm 9.8$ & $81.5 \pm 9.9$ & $81.1 \pm 9.1$ & n.s. & $81.4 \pm 11.0$ & $81.3 \pm 9.6$ & n.s. \\
\hline Diabetes (n/\%) & $71 / 20.2$ & $42 / 21.2$ & $22 / 31.0$ & $3 / 4.9$ & $4 / 18.2$ & 0.003 & $63 / 20.7$ & $8 / 16.7$ & n.s. \\
\hline CAD (n/\%) & 73 / 20.7 & 49 / 24.7 & $20 / 28.2$ & $3 / 4.9$ & $1 / 4.5$ & 0.001 & $59 / 19.4$ & $14 / 29.2$ & n.s. \\
\hline
\end{tabular}

$\mathrm{CMV}=$ cytomegaly virus, $\mathrm{HD}=$ hemodialysis, $\mathrm{PD}=$ peritoneal dialysis, $\mathrm{BP}=$ blood pressure, $\mathrm{CAD}=$ documented coronary artery disease

$\mathrm{ABO}_{\mathrm{c}}=\mathrm{ABO}$-compatible, $\mathrm{ABO} \mathrm{O}_{\mathrm{i}}=\mathrm{ABO}$-incompatible. 
Table 2 Transplant relevant data

\begin{tabular}{|c|c|c|c|c|c|c|c|c|c|}
\hline Variable & $\begin{array}{l}\text { All patients } \\
(\mathrm{n}=352)\end{array}$ & $\begin{array}{c}\text { Deceased <65y } \\
(n=198)\end{array}$ & $\begin{array}{c}\text { Deceased >65y } \\
\quad(n=71)\end{array}$ & $\begin{array}{l}\text { Living } \mathrm{ABO}_{\mathrm{c}} \\
\quad(\mathrm{n}=61)\end{array}$ & $\begin{array}{l}\text { living } \mathrm{ABO}_{i} \\
\quad(\mathrm{n}=22)\end{array}$ & $\begin{array}{l}\text { ANOVA or } \\
\text { chi }^{2} p \text {-value }\end{array}$ & $\begin{array}{c}\text { No BK } \\
(n=304)\end{array}$ & $\begin{array}{c}\text { BK } \\
(n=48)\end{array}$ & $\begin{array}{c}\text { T-test or } \\
\text { chi }^{2} p \text {-value }\end{array}$ \\
\hline Donor age (years) & $54.5 \pm 14.0$ & $48.3 \pm 13.0$ & $70.9 \pm 5.7$ & $55.9 \pm 8.6$ & $52.4 \pm 8.4$ & 0.0002 & $54.1 \pm 13.8$ & $56.8 \pm 15.1$ & n.s. \\
\hline Donor sex (male / female) & $166 / 186$ & $95 / 103$ & $33 / 38$ & $29 / 32$ & $9 / 13$ & n.s. & $146 / 158$ & $20 / 28$ & n.s. \\
\hline Donor BMI $\left(\mathrm{kg} / \mathrm{m}^{2}\right)$ & $26.5 \pm 4.5$ & $26.6 \pm 4.5$ & $27.1 \pm 5.5$ & $26.2 \pm 3.4$ & $25.1 \pm 3.2$ & n.s. & $26.4 \pm 4.3$ & $27.4 \pm 5.3$ & n.s. \\
\hline Donor creatinine (mg/dl) & $0.98 \pm 0.62$ & $1.03 \pm 0.75$ & $1.01 \pm 0.53$ & $0.81 \pm 0.16$ & $0.85 \pm 0.12$ & n.s. & $0.99 \pm 0.65$ & $0.91 \pm 0.34$ & n.s. \\
\hline Donor creatinine >1.5 mg/dl (n/\%) & $37 / 10.5$ & $26 / 13.1$ & $11 / 15.5$ & $0 / 0$ & $0 / 0$ & 0.004 & $33 / 10.9$ & $4 / 8.3$ & n.s. \\
\hline Donor diuresis $(\mathrm{ml} / \mathrm{h})$ & $156 \pm 99$ & $177 \pm 106$ & $158 \pm 101$ & $92 \pm 24$ & $108 \pm 33$ & 0.0005 & $158 \pm 101$ & $144 \pm 82$ & n.s. \\
\hline Cold ischemic time (h) & $10.3 \pm 5.8$ & $13.3 \pm 4.1$ & $11.2 \pm 3.9$ & $2.2 \pm 1.2$ & $2.6 \pm 1.1$ & 0.0006 & $10.2 \pm 5.8$ & $10.7 \pm 5.8$ & n.s. \\
\hline HLA mismatch (n) & $2.9 \pm 1.7$ & $2.4 \pm 1.6$ & $3.9 \pm 1.2$ & $3.4 \pm 1.6$ & $3.4 \pm 1.3$ & 0.0001 & $2.9 \pm 1.6$ & $2.9 \pm 1.7$ & n.s. \\
\hline Number of 0 mismatches $(\mathrm{n} / \%)$ & $41 / 11.6$ & $38 / 19.2$ & $1 / 1.4$ & $2 / 3.3$ & $0 / 0$ & 0.0004 & $34 / 11.2$ & $7 / 14.6$ & n.s. \\
\hline Tacrolimus (n/\%) & $280 / 79.5$ & $163 / 82.3$ & $45 / 63.4$ & $50 / 82.0$ & $22 / 100$ & 0.0005 & $242 / 79.6$ & $37 / 77.1$ & n.s. \\
\hline Cyclosporine (n/\%) & $72 / 20.5$ & $35 / 17.7$ & $26 / 36.6$ & $11 / 18.0$ & $0 / 0$ & 0.0005 & $62 / 20.4$ & $11 / 22.9$ & n.s. \\
\hline ATG-induction (n/\%) & $65 / 18.5$ & $48 / 24.2$ & $8 / 11.3$ & $7 / 11.5$ & $2 / 9.1$ & 0.018 & $57 / 18.7$ & $8 / 16.7$ & n.s. \\
\hline IL2-Induction (n/\%) & $280 / 79.5$ & $145 / 73.3$ & $61 / 85.9$ & $54 / 88.5$ & $20 / 90.9$ & 0.010 & $241 / 79.3$ & $39 / 81.2$ & n.s. \\
\hline No induction (n/\%) & $7 / 2.0$ & $5 / 2.5$ & $2 / 2.8$ & $0 / 0$ & $0 / 0$ & n.s. & $6 / 2.0$ & $1 / 2.1$ & n.s. \\
\hline Early steroid withdrawal (n/\%) & $46 / 13.1$ & $30 / 15.1$ & $12 / 16.9$ & $4 / 6.6$ & $0 / 0$ & n.s. & $43 / 14.1$ & $3 / 6.2$ & n.s. \\
\hline PRAs (n/\%) & $45 / 12.8$ & $34 / 17.2$ & $6 / 8.4$ & $4 / 6.6$ & $1 / 4.5$ & 0.044 & $36 / 11.8$ & $9 / 18.7$ & n.s. \\
\hline$C M V$ risk profile $\left(D^{-} R^{-}, D^{-} R^{+}, D^{+} R^{+}, D^{+} R^{-}\right) \%$ & $18 / 26 / 35 / 21$ & $18 / 32 / 30 / 20$ & $10 / 25 / 44 / 21$ & $21 / 15 / 38 / 26$ & $27 / 5 / 41 / 27$ & 0.035 & $18 / 23 / 36 / 23$ & $15 / 44 / 31 / 10$ & 0.014 \\
\hline Study participant (n/\%) & $126 / 35.8$ & $82 / 41.4$ & $22 / 31.0$ & $22 / 36.1$ & $0 / 0$ & 0.001 & $117 / 38.5$ & $9 / 18.7$ & 0.009 \\
\hline Primary function (n/\%) & $251 / 71.3$ & $128 / 64.6$ & $47 / 66.2$ & $55 / 90.2$ & $21 / 95.5$ & 0.0006 & $216 / 71.1$ & $35 / 72.9$ & n.s. \\
\hline Creatinine at discharge $(\mathrm{mg} / \mathrm{dl})$ & $2.04 \pm 0.94$ & $2.08 \pm 0.98$ & $2.40 \pm 1.00$ & $1.67 \pm 0.69$ & $1.56 \pm 0.50$ & 0.0001 & $2.00 \pm 0.86$ & $2.28 \pm 1.33$ & 0.057 \\
\hline eGFR at discharge $(\mathrm{ml} / \mathrm{min})$ & $35.5 \pm 14.7$ & $35.1 \pm 14.8$ & $27.3 \pm 9.8$ & $43.1 \pm 14.6$ & $42.8 \pm 13.1$ & 0.0001 & $35.9 \pm 14.7$ & $33.3 \pm 14.4$ & n.s. \\
\hline
\end{tabular}

BMI = body mass index, GFR = glomerular filtration rate, ATG = antithymocyte globulin, IL2 = interleukin 2, PRA = panel reactive antibodies, CMV = cytomegalovirus. 

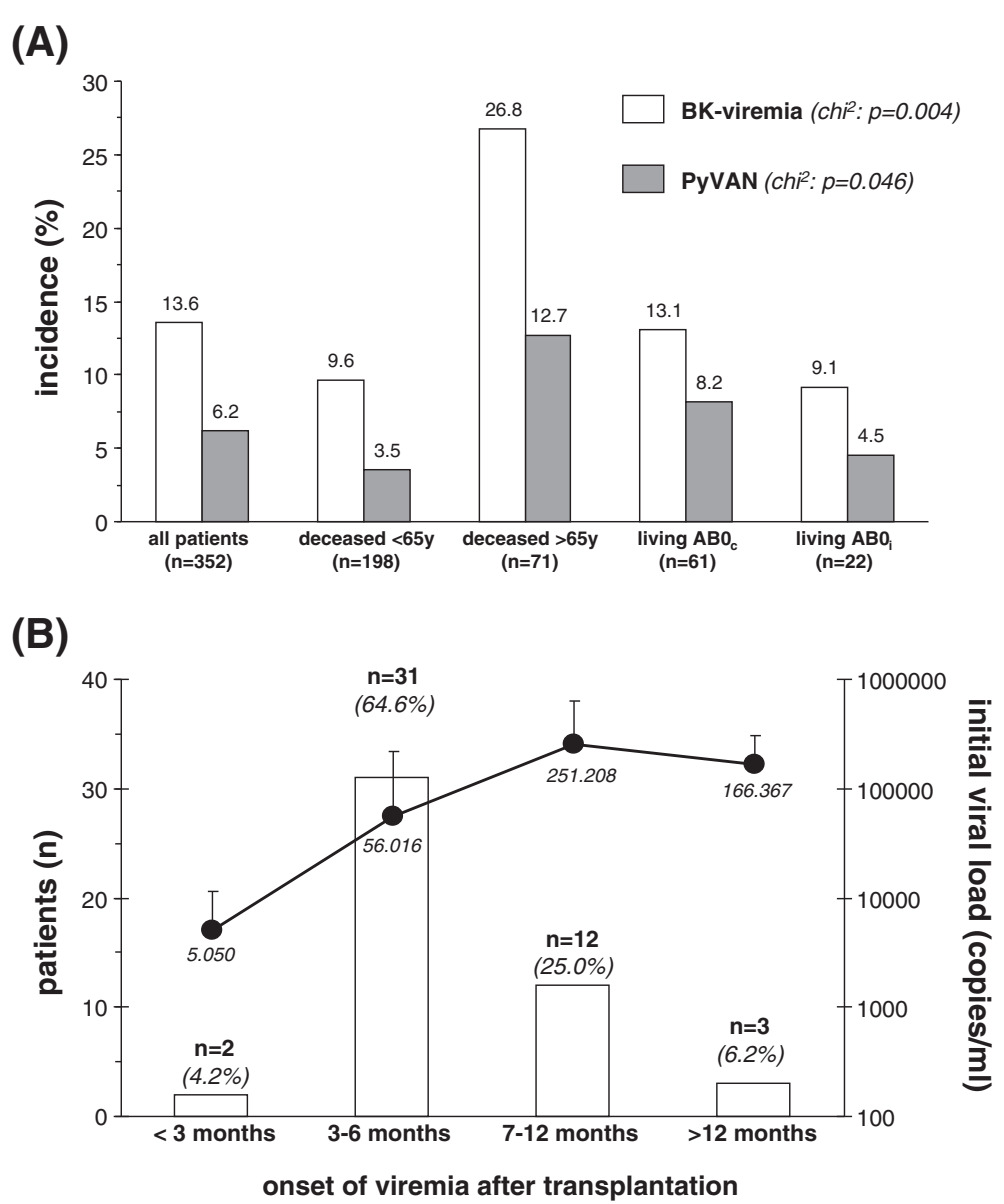

Figure 1 Incidence of BK-viremia and PyVAN in the entire cohort and subgroups (Figure 1A). Time of onset of BK-viremia and corresponding viral load (Figure 1B).

mismatch, donor and recipient sex and donor age were not associated with BK viremia.

All variables that were significant in univariate analyses as well as the above mentioned known risk factors were entered into the multivariate model. In the multivariate logistic regression analysis the following variables remained significant predictors for BK viremia: lack of participation in a prospective clinical transplant trial, BPAR, previous transplantation, and donor (IgG-) as well as recipient (IgG+) CMV-serostatus (Table 4).

Interestingly, patients who were enrolled into a prospective clinical trial were less likely to develop BK viremia (Table 2). Thus, the incidence of BK viremia was $17.3 \%$ $(39 / 226)$ in non-study participants and $7.1 \%(9 / 126)$ in patients recruited for a clinical trial $(\mathrm{p}=0.009)$. Whereas the frequency of induction therapy with ATG or basiliximab did not differ between study versus non-study participants, use of CyA as baseline CNI was more frequent in patients enrolled into a clinical trial (38/126 or $30.2 \%$ vs. $35 / 226$ or $15.5 \%, \mathrm{p}=0.002)$. More intriguingly, 36 of the 38 patients initially treated with cyclosporine as baseline CNI were enrolled into the HERAKLES trial (ClinicalTrials.gov NCT00514514), a trial in which standard CNI therapy with CyA was compared to a low dose CNI, or CNI free immunosuppressive regimen. None of these 36 patients developed BK viremia. Notably, screening intervals for BK viral replication did not differ between study versus nonstudy participants.

Patients with BK viremia were more likely to have a prior episode of BPAR within the first year after transplantation than patients with absence of viral replication (39.6\% vs. $23.0 \%$ BPAR within the first year, $\mathrm{p}=0.014)$. As previously shown by others, patients with previous renal transplants had a higher incidence of BK viremia (Table 1).

Donor (IgG-) and recipient (IgG+) CMV serostatus were associated with BK viremia. The lowest incidence of BK viremia was seen in high $C M V$ risk patients $\left(D^{+} / R^{-}: 5 / 76\right.$ or $6.6 \%$ ), followed by low CMV risk patients ( $D^{-} / R^{-}: 7 / 62$ or $11.3 \%)$. The highest incidence was noted in intermediate $C M V$ risk patients $\left(D^{+} / R^{+}: 15 / 123\right.$ or $12.2 \% ; D^{-} / R^{+}: 21 /$ 91 or $23.1 \%, p=0.014$, Figure $3 \mathrm{~A})$. 
(A)

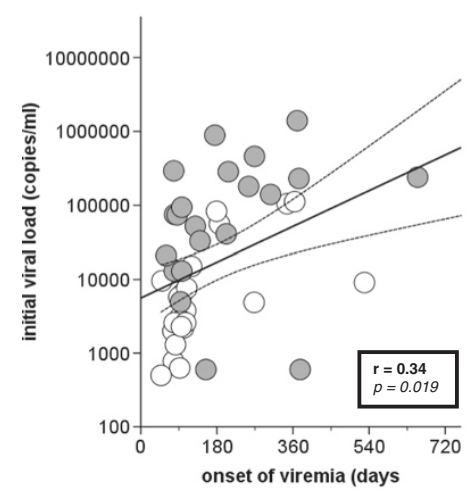

BK-viremia $(\mathbf{n}=26)$

PyVAN (n=22)
(B)

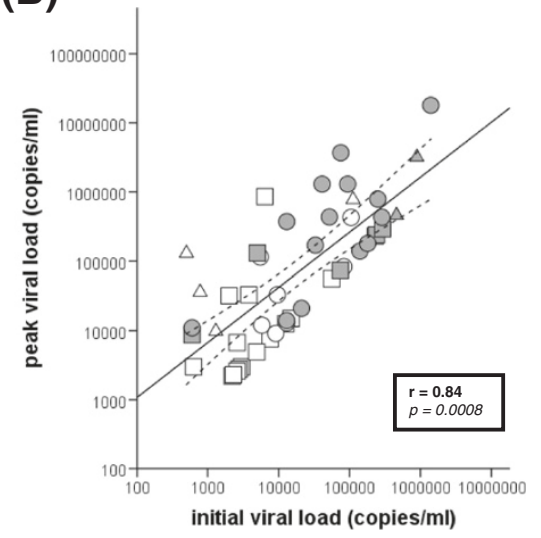

BK viremia: reduction of immunosuppression $(n=15)$

PyVAN: reduction of immunosuppression $(n=7)$

BK viremia: conversion other than low CyA + mTORi $(n=4)$

PyVAN: conversion other than low CyA + mTORi $(n=2)$

BK viremia: conversion low CyA + mTORi $(n=7)$

PyVAN: conversion low CyA + mTORi $(n=13)$

Figure 2 Scatterplot showing correlation between onset of BK-viremia and initial viral load (Figure 2A). Correlation of initial and peak viral load in patients with BK viremia and PyVAN (open vs. grey symbols) under different treatment strategies (Figure 2B).

During study follow-up 52 of 352 patients (14.8\%) experienced CMV replication of at least 1000 copies $/ \mathrm{ml}$. The incidence of CMV infection in patients with $\mathrm{BK}$ viremia was $6 / 48$ or $12.5 \%$.

\section{Graft function and outcome of patients with BK Viremia and PyVAN}

During follow-up 5 of 48 patients (10.4\%) with a history of PyVAN lost their allograft. In three of these patients graft loss was clearly related to PyVAN, another patient lost his kidney due to ongoing antibody mediated rejection and PyVAN. The fifth patient received an ECD kidney and developed low level viremia, graft loss occurred $\sim 5$ months after viral clearance and a final biopsy showed resolution of PyVAN.

Duration of viral clearance was prolonged in patients with PyVAN versus patients with BK viremia only (267 vs. 135 days, $\mathrm{p}=0.018$ ). A total of $\mathrm{n}=5$ patients still have ongoing low level viremia (see Additional file 1: Table S1).

In patients with negative screening for BK renal allograft function improved within the first year after transplantation whereas in patients with BK viremia and/or PyVAN graft function did not improve during this time period (Figure 3B). Within the 26 patients with BK viremia without PyVAN treatment consisted of reduction of immunosuppression $(\mathrm{n}=15)$, conversion to low CyA plus mTORi

Table 3 Univariate binary logistic regression analysis; variables associated with BK viremia

\begin{tabular}{lccccc}
\hline Variable & $\boldsymbol{\beta}$ & S.E. & OR & $\mathbf{9 5 \%}$ Cl & p-value \\
\hline ESP recipient status (yes vs. no) & 1.17 & 0.33 & 3.23 & $1.70-6.16$ & 0.0003 \\
Recipient age (per year) & 0.040 & 0.013 & 1.04 & $1.01-1.07$ & 0.002 \\
BPAR (yes vs. no) & 0.96 & 0.34 & 2.60 & $0.33-5.08$ & $0.17-0.79$ \\
Study participant (yes vs. no) & -1.00 & 0.39 & 0.37 & $0.060-0.84$ & 0.010 \\
Mode of RRT (vs. HD) & -1.49 & 0.67 & 0.22 & $1.08-3.72$ & 0.027 \\
Donor CMV IgG negative (yes vs. no) & 0.70 & 0.31 & $1.07-3.29$ & 0.027 \\
Previous transplantation (vs. first) & 0.63 & 0.29 & 2.12 & $1.06-4.24$ & 0.028 \\
Recipient CMV IgG positive (yes vs. no) & 0.75 & 0.004 & 1.009 & $1.000-1.018$ & 0.033 \\
LDL cholesterol (per mg/dl) & 0.009 & 0.002 & 1.004 & $1.000-1.009$ \\
25-hydroxy vitamin D level (per nmol/l) & 0.004 & & 0.041
\end{tabular}

$\mathrm{ESP}=$ European Senior Program, BPAR = biopsy proven acute rejection, $\mathrm{RRT}=$ renal replacement therapy, $\mathrm{OR}=$ odds ratio, $\mathrm{Cl}=$ confidence interval. 
Table 4 Multivariate logistic regression analysis; predictors of BK viremia

\begin{tabular}{lccccc}
\hline Variable & $\boldsymbol{\beta}$ & S.E. & OR & 95\% Cl & p-value \\
\hline Study participant (yes vs. no) & -1.82 & 0.56 & 0.16 & $0.054-0.49$ & 0.001 \\
BPAR (yes vs. no) & 1.33 & 0.47 & 3.79 & $1.50-9.58$ & 0.005 \\
Donor CMV IgG negative (yes vs. no) & 1.00 & 0.45 & 2.71 & $1.13-6.51$ & 0.026 \\
Recipient CMV IgG positive (yes vs. no) & 1.05 & 0.49 & 2.84 & $1.10-7.37$ & 0.031 \\
Previous transplantation (vs. first) & 1.009 & 0.49 & 2.74 & $1.05-7.15$ & 0.039 \\
\hline
\end{tabular}

For multivariate analyses all parameters that were significant in the univariate logistic regression model as well as the following known risk factors for BK viremia that were negative in the univariate approach were entered into the model: recipient as well as donor sex, donor age, use of CNI (tacrolimus or CyA), ATG, HLA mismatch).

$(n=7)$ or to other regimens $(n=4)$. Out of the 22 patients with PyVAN the majority was converted to low CyA plus mTORi $(n=13)$ or other regimes $(n=2)$, while the remainder where treated with reduced doses of their original immunosuppressants $(n=7)$.
In patients with BK viremia without PyVAN renal function remained stable within the first year after transplantation irrespective of selected treatment (Figure 4A + B). In contrast, in patients with PyVAN renal function deteriorated over time (Figure $4 C+D$ ). In this group of patients,

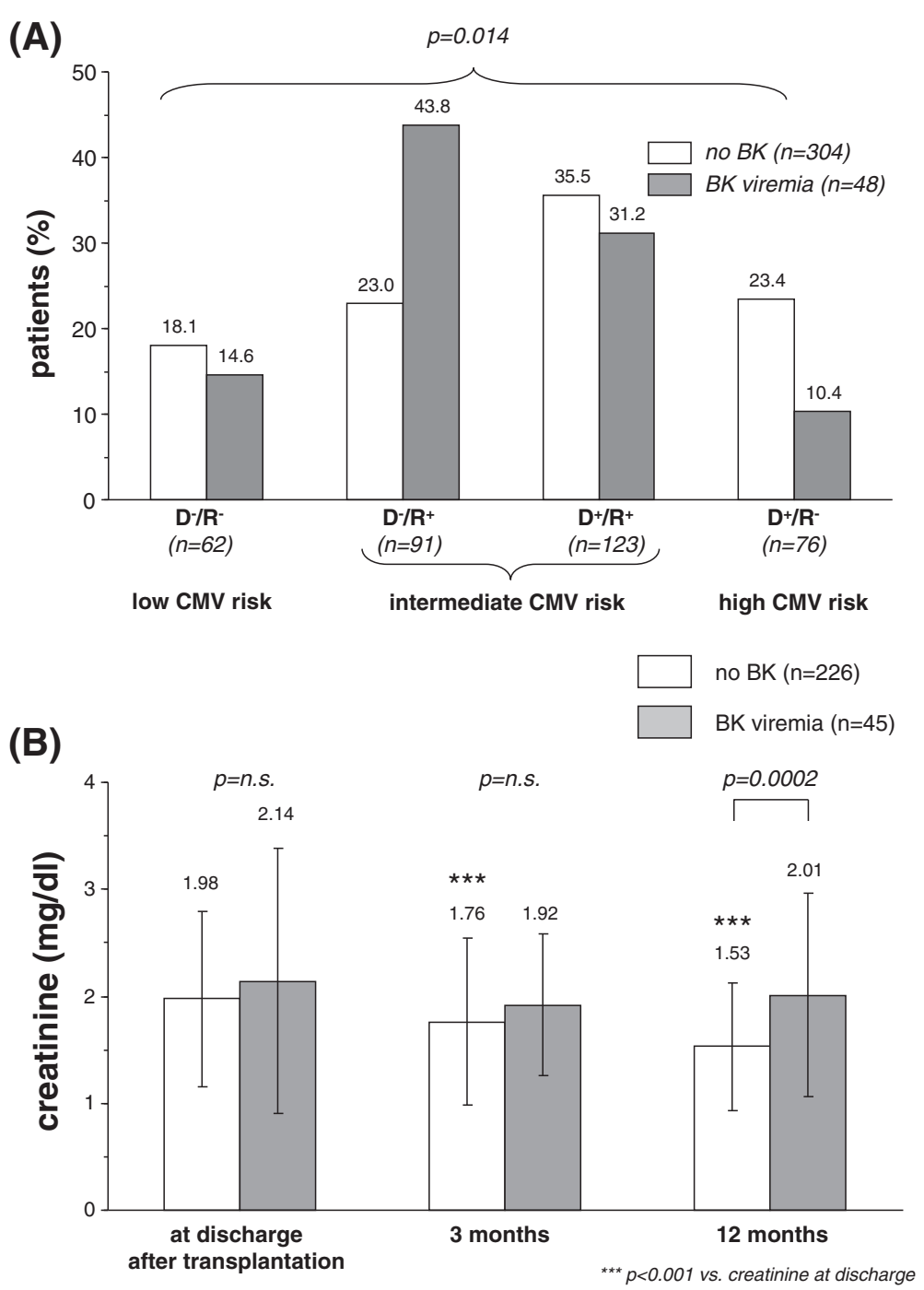

Figure 3 Donor/Recipient CMV risk profiles in patients with (grey bars) and without BK-viremia (white bars, Figure $3 \mathrm{~A}$ ). Creatinine course at the time of discharge as well as 3 and 12 months after transplantation in patients with (grey bars) and without BK-viremia (white bars; Figure 3B). Only patients with complete creatinine values at all time points were included. 


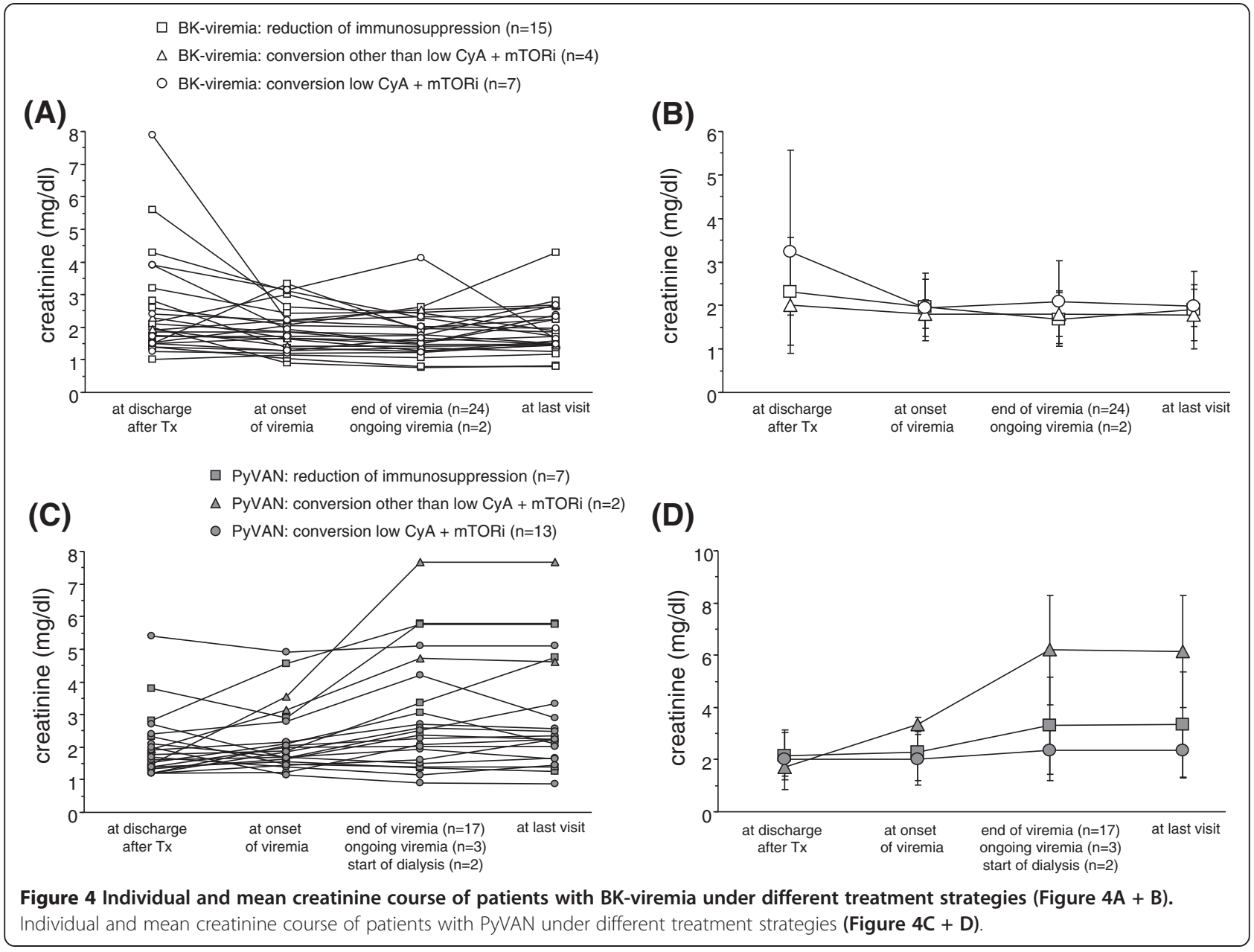

conversion of immunosuppression to a low CyA plus mTORi based regimen tended to be associated with a better short-term renal outcome compared to patients treated with reduction of existing immunosuppression. Duration of viremia, peak viral load as well as change of creatinine and albuminuria between onset and clearance of BK viremia did not differ between the three treatment groups (Figure 5A-D).

Overall, reduction or conversion of immunosuppression was relatively safe and well tolerated. Three patients with BK viremia but lack of PyVAN developed donor specific antibodies with biopsy proven antibody mediated rejection during follow-up. These patients were treated with rituximab and a series of immunoadsorption without relapse of BK-viral infection. In two of these patients, in which immunosuppression had been initially reduced to overcome BK viremia, dose adjustment of triple immunosuppressive regimen was performed whereas the third patient that had been switched to low dose CyA plus mTORi was converted to tacrolimus plus mycophenolate mofetil.

During follow-up one patient treated with reduction of immunosuppression to achieve viral clearance later died from sepsis due to pneumocystis pneumonia (see Additional file 1: Table S1). Another patient treated with a combination of tacrolimus and sirolimus developed severe Legionella pneumonia requiring intensive care treatment.

\section{Discussion}

The novel findings of this retrospective cohort study include the identification of putative novel risk factors for BK viral infection after kidney transplantation and the analysis of conversion to a low CyA plus mTORi based immunosuppressive regimen especially in patients with biopsy proven PyVAN. To the best of our knowledge, the latter observation, although mainly hypothesis generating and seeking for confirmation in future clinical trials, has not yet been addressed by others.

Overall, incidence rates of BK viremia (13.6\%) and PyVAN (6.2\%) in our study were similar to previously published data [7,16-18]. However, we observed a rather high incidence of BK viremia and PyVAN especially in old transplant recipients. Since both donor and recipient age are known risk factors for BK-viral replication, we 


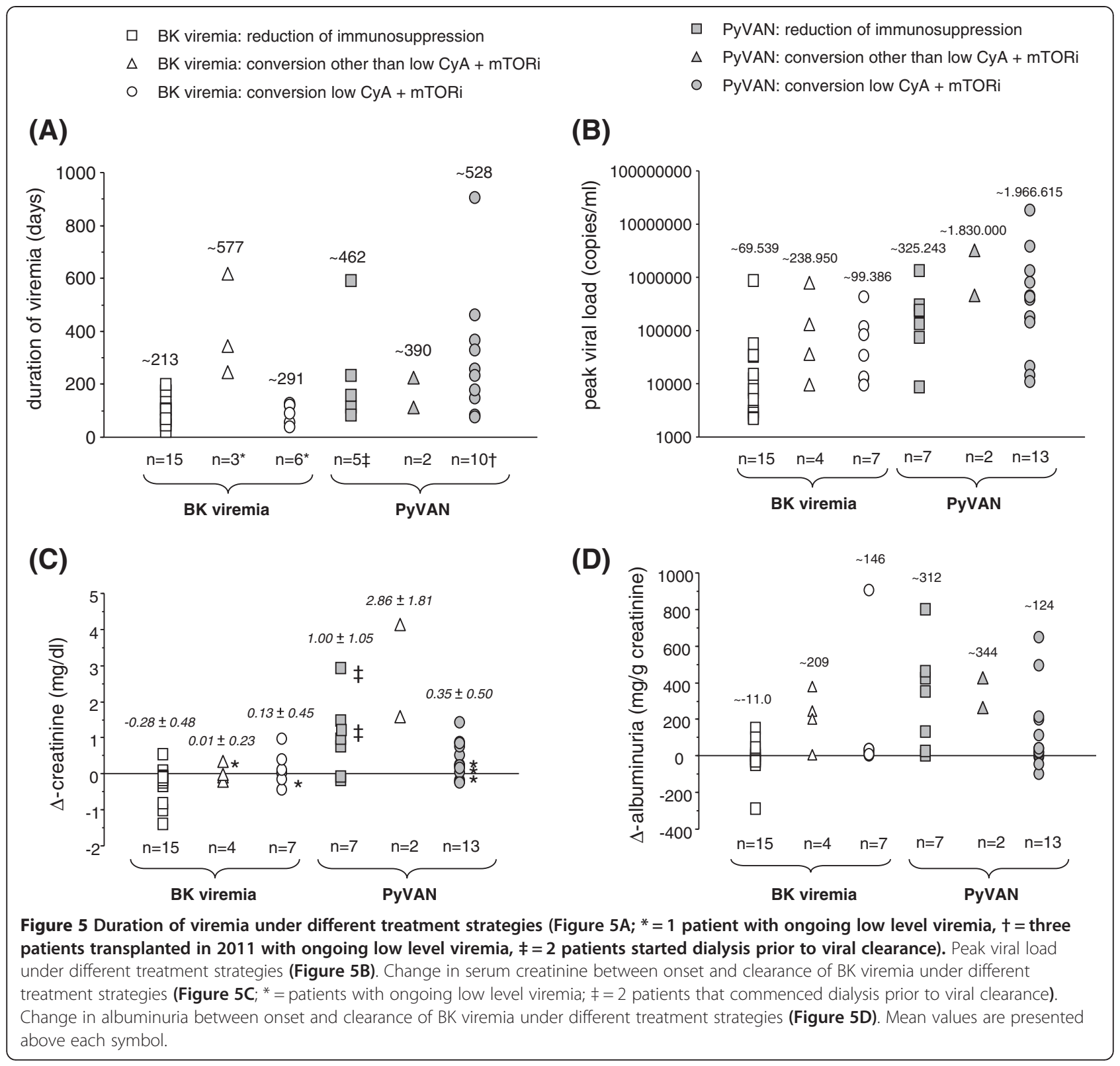

observed the highest incidence of viral infection in our ESP allograft recipients, although patients in this subgroup were more likely to receive CyA as initial CNI for which lower rates of BK viral infection have been reported in the literature as compared to tacrolimus [19]. One explanation for this discrepancy maybe the higher incidence of BPAR observed in CyA treated patients in our study cohort. In fact, BPAR emerged as a risk factor for BK viral infection and vice versa. This fateful relationship reflects the current dilemma in the management of patients that are at risk for BPAR or BK viral infection.

Interestingly, the incidence of $\mathrm{BK}$ viremia in $\mathrm{ABO}$ incompatible living donation was lower than in AB0compatible transplantation, although others have reported controversial findings [20,21]. We and others have recently described plasma cell infiltrates in renal allografts of patients with PyVAN suggesting that humoral immunity may play a role in polyoma viral disease [22,23], and it is intriguing to speculate that the use of rituximab may modulate the risk for PyVAN. However, in our study neither the use of ATG or rituximab given within the first year after transplantation was associated with BK viremia. In line with this observation polyoma virus replication was not associated with rituximab therapy in pediatric patients with nephrotic syndrome [24].

In agreement with previous observations recipient age, BPAR and previous transplantation emerged as strong risk factors for BK viremia [25-27]. Other known risk factors 
such as male gender, HLA mismatch, use of tacrolimus as $\mathrm{CNI}$ and induction therapy or later use of ATG were not associated with a greater risk of BK viral infection in our study cohort. In addition, to these known variables we identified novel putative risk factors for BK viremia. Thus, patients that were enrolled into a clinical transplant trial were at markedly lower risk to develop BK viremia. The impact of study participation most likely reflects a greater focus on target levels of immunosuppression and to some extent the greater use of CyA as initial CNI, although the effect remained significant in multivariate analyses accounting for baseline immunosuppression and recipient age.

Another striking observation was that both negative donor and positive recipient serostatus for CMV emerged as predictors for BK virus infection. Accordingly, the highest incidence of BK viremia was observed in CMV seropositive patients that received an allograft from a seronegative donor, whereas the lowest incidence was seen in CMV high risk patients, i.e. donor CMV seropositive and recipient CMV seronegative. While co-infection of polyomavirus and cytomegalovirus have been reported in renal transplant recipients $[28,29]$ and after stem cell transplantation [30], the impact of recipient CMV seropositivity without evidence of CMV viremia on polyomavirus infection is unknown. However, in a study of 132 hematopoietic stem cell transplant recipients a positive recipient CMV serostatus and the underlying disease emerged as the only risk factors associated with BK viremia [31].

A negative donor serostatus for CMV was only associated with a markedly greater risk to develop BK infection if allografts were transplanted into seropositive recipients. Thus, $\mathrm{D}^{-} \mathrm{R}^{+} \mathrm{CMV}$ serostatus may trigger an immune response within the CMV naïve allograft that may predispose to other opportunistic viral infections. This hypothesis needs to be further investigated. Overall, the effect of CMV serostatus on BK viral infection is unlikely a chance finding and cannot be explained by the different usage of CMV prophylaxis with valganciclovir given the low incidence of $\mathrm{BK}$ in $\mathrm{D}^{-} \mathrm{R}^{-}$patients.

Another interesting finding was that all but one patient with BK viremia were treated with hemodialysis prior to transplantation. Since peritoneal dialysis and preemptive patients only reflected $17 \%$ of the entire study cohort this could indeed be a chance finding. Possible explanations that may otherwise explain this observation could be an altered immune system in patients treated with an extracorporeal renal replacement therapy and better preserved residual diuresis in peritoneal dialysis and pre-emptive transplant candidates.

Our strategy in the management of patients with BK viremia and PyVAN, namely reduction or conversion of immunosuppression resulted in a favourable outcome in most patients. In patients with BK viremia without evidence of PyVAN reduction of net immunsuppression led to rapid viral clearance and conversion of immunosuppression offered no benefit. Switch of immunosuppression to a low CyA plus mTORi based regimen in patients with biopsy proven PyVAN was safe, well tolerated and noninferior to reduction of immunosuppression with respect to short-term follow-up. To the best of our knowledge the combination of low dose CyA and mTORi has not yet been studied in a comparable size of patients.

However, the role of mTORi in the treatment of BK viral infection has gained more attention within recent years. Available data suggests that mTORi reduce the expression of BK virus large $\mathrm{T}$ antigen and antigen-dependent $\mathrm{T}$-cell expansion in a dose dependent manner [32,33]. In line with these observations renal transplant recipients treated with a mTORi based immunosuppressive regimen display BK viral infection rates at the lower end reported in the literature. In a retrospective cohort of comparable size in which all patients $(n=344)$ received sirolimus the incidence of BK viremia was only $1.7 \%$ [34]. Until now mTORi has largely been used as rescue therapy in patients in whom other strategies were ineffective or failed [35-38].

Our study is limited due to its retrospective, single centre design with lack of long-term follow-up. In addition, protocol biopsies and sampling of blood for BK viremia was not consistently available for all patients. Therefore, our findings of selected treatments to achieve viral clearance are hypothesis generating and need confirmation in prospective clinical trials in which changes of immunosuppression are not dictated by immunological risk. Nevertheless, our data indicate that conversion of immunosuppression to a low CNI plus mTORi based immunosuppressive regimen is feasible and safe.

\section{Conclusion}

In conclusion, this retrospective cohort study highlights the importance of active surveillance for BK viral replication following kidney transplantation especially in aged transplant recipients. In addition to previously known risk factors, we identified novel risk factors for BK viral infection that need to be confirmed in future clinical trials. In patients with biopsy proven PyVAN conversion of immunosuppression to a low CyA / mTORi based regimen showed promising results that warrant further investigation in future trials.

\section{Additional file}

Additional file 1: Table S1. Characteristics of patients with BK viremia and BK nephropathy sorted by day of detection of viremia.

\footnotetext{
Abbreviations

ATG: Antilymphocyte globulin; $A B 0_{c}$ : $A B O$ compatible transplant; $A B 0_{i}: A B O$ incompatible transplant; BK: Polyoma virus; BPAR: Biopsy proven acute rejection; CMV: Cytomegaly virus; CNI: Calcineurin inhibitor; CyA: Cyclosporine A: ECD: Expanded criteria donor; mTOR: Mammalian target of rapamycin;
} 
PCR: Polymerase chain reaction; PyVAN: Polyoma virus BK nephropathy; RCT: Randomized clinical trial; SV40: Simian virus 40.

\section{Competing interests}

This study was investigator-initiated. Dr. Jacobi reports receiving grant support for other projects and lecture fees from Novartis. Since 2012 Novartis provides financial support for the maintenance and data entry of the transplant database, but Novartis had no impact on data acquisition and interpretation. Dr. May, who is employed by Novartis, served as consultant advisor for the statistical analyses.

\section{Authors' contributions}

This retrospective analysis was initiated by $\mathrm{J}$ who wrote the manuscript. Maintenance of the transplant database was provided by JJ, AP and AW. MB, JV and KUA performed histological analyses of transplant biopsies, KK and AK were responsible for PCR screening for BK viral replication. $\mathrm{KFH}, \mathrm{KH}, \mathrm{BW}$, KUE and KUA were critically involved in data interpretation and drafting of the manuscript. CM served as consultant advisor for statistical analysis. The manuscript was approved by all authors. All authors read and approved the final manuscript.

\section{Acknowledgments}

We are indebted to Antje Weixelbaum and Dr. Sabine Jank for recruiting patients for follow-up visits.

\section{Author details}

${ }^{1}$ Department of Nephrology and Hypertension, Friedrich-Alexander-University Erlangen-Nürnberg, Ulmenweg 18, 91054, Erlangen, Germany. 'Department of Nephropathology, Friedrich-Alexander-University Erlangen-Nürnberg, Nuremberg, Germany. ${ }^{3}$ Institute of Clinical and Molecular Virology, Friedrich-Alexander-University Erlangen-Nürnberg, Nuremberg, Germany. ${ }^{4}$ Department of Urology, Friedrich-Alexander-University Erlangen-Nürnberg, Nuremberg, Germany. ${ }^{5}$ Biometrics Department, Novartis Pharma, Nuremberg, Germany.

Received: 8 April 2013 Accepted: 30 September 2013

Published: 2 October 2013

\section{References}

1. Ekberg H, Tedesco-Silva H, Demirbas A, Vitko S, Nashan B, Gurkan A, et al: Reduced exposure to calcineurin inhibitors in renal transplantation. N Engl J Med 2007, 357(25):2562-2575. Epub 2007/12/21.

2. Vincenti F, Larsen C, Durrbach A, Wekerle T, Nashan B, Blancho G, et al: Costimulation blockade with belatacept in renal transplantation. $N$ Engl J Med 2005, 353(8):770-781. Epub 2005/08/27.

3. Hirsch HH, Steiger J: Polyomavirus BK. Lancet Infect Dis 2003, 10:611-623. Epub 2003/10/03.

4. Gardner SD, Field AM, Coleman DV, Hulme B: New human papovavirus (B.K.) isolated from urine after renal transplantation. Lancet 1971, 1(7712):1253-1257.

5. Kean JM, Rao S, Wang M, Garcea RL: Seroepidemiology of human polyomaviruses. PLoS Pathog 2009, 5(3):e1000363. Epub 2009/03/28

6. Stolt A, Sasnauskas K, Koskela P, Lehtinen M, Dillner J: Seroepidemiology of the human polyomaviruses. J Gen Virol 2003, 84(Pt 6):1499-1504. Epub 2003/05/29

7. Hirsch HH, Knowles W, Dickenmann M, Passweg J, Klimkait T, Mihatsch MJ, et al: Prospective study of polyomavirus type BK replication and nephropathy in renal-transplant recipients. N Engl J Med 2002, 347(7):488-496. Epub 2002/08/16.

8. Hussain S, Bresnahan BA, Cohen EP, Hariharan S: Rapid kidney allograft failure in patients with polyoma virus nephritis with prior treatment with antilymphocyte agents. Clin Transplant 2002, 16(1):43-47. Epub 2002/05/02.

9. Ramos E, Drachenberg CB, Papadimitriou JC, Hamze O, Fink JC, Klassen DK, et al: Clinical course of polyoma virus nephropathy in 67 renal transplant patients. J Am Soc Nephrol 2002, 13(8):2145-2151. Epub 2002/07/26.

10. Dall A, Hariharan S: BK virus nephritis after renal transplantation. Clin J Am Soc Nephrol 2008, 3(Suppl 2):S68-S75. Epub 2008/03/20.

11. Thomas A, Dropulic LK, Rahman MH, Geetha D: Ureteral stents: a novel risk factor for polyomavirus nephropathy. Transplantation 2007, 84(3):433-436. Epub 2007/08/19.

12. Schaub S, Hirsch HH, Dickenmann M, Steiger J, Mihatsch MJ, Hopfer H, et al: Reducing immunosuppression preserves allograft function in presumptive and definitive polyomavirus-associated nephropathy. Am $\mathrm{J}$ Transplant 2010, 10(12):2615-2623. Epub 2010/12/01.

13. Kasiske BL, Zeier MG, Chapman JR, Craig JC, Ekberg H, Garvey CA, et al: KDIGO clinical practice guideline for the care of kidney transplant recipients: a summary. Kidney Int 2010, 77(4):299-311. Epub 2009/10/23.

14. Sar A, Worawichawong S, Benediktsson H, Zhang J, Yilmaz S, Trpkov K: Interobserver agreement for Polyomavirus nephropathy grading in renal allografts using the working proposal from the 10th Banff Conference on Allograft Pathology. Hum Pathol 2011, 42(12):2018-2024. Epub 2011/07/08.

15. Kuypers DR: Management of polyomavirus-associated nephropathy in renal transplant recipients. Nat Rev Nephrol 2012, 8(7):390-402. Epub 2012/04/18.

16. Brennan DC, Agha I, Bohl DL, Schnitzler MA, Hardinger KL, Lockwood M, et al: Incidence of BK with tacrolimus versus cyclosporine and impact of preemptive immunosuppression reduction. Am J Transplant 2005, 5(3):582-594. Epub 2005/02/15.

17. Koukoulaki M, Grispou E, Pistolas D, Balaska K, Apostolou T, Anagnostopoulou $\mathrm{M}$, et al: Prospective monitoring of BK virus replication in renal transplant recipients. Transpl Infect Dis 2009, 11(1):1-10. Epub 2008/09/25

18. Yeo FE, Yuan CM, Swanson SJ, Reinmuth B, Kiandoli LC, Kaplan KJ, et al: The prevalence of BK polyomavirus infection in outpatient kidney transplant recipients followed in a single center. Clin Transplant 2008, 22(5):532-541. Epub 2008/07/25

19. Hirsch HH, Vincenti F, Friman S, Tuncer M, Citterio F, Wiecek A, et al: Polyomavirus BK Replication in De Novo Kidney Transplant Patients Receiving Tacrolimus or Cyclosporine: A Prospective, Randomized, Multicenter Study. Am J Transplant 2012. Epub 2012/11/10.

20. Sharif A, Alachkar N, Bagnasco S, Geetha D, Gupta G, Womer K, et al: Incidence and Outcomes of BK Virus Allograft Nephropathy among ABO- and HLA-Incompatible Kidney Transplant Recipients. Clin J Am Soc Nephrol 2012, 7(8):7-1320. Epub 2012/05/26.

21. Habicht A, Broker V, Blume C, Lorenzen J, Schiffer M, Richter N, et al: Increase of infectious complications in ABO-incompatible kidney transplant recipients-a single centre experience. Nephrol Dial Transplant 2011, 26(12):4124-31. Epub 2011/05/31.

22. Buettner $M, X u H$, Bohme $R$, Seliger $B$, Jacobi J, Wiesener $M$, et al: Predominance of Th2 cells and plasma cells in polyoma virus nephropathy: a role for humoral immunity? Hum Pathol 2012, 43(9):1453-62. Epub 2012/03/13.

23. Kemeny $E_{1}$ Hirsch HH, Eller J, Durmuller U, Hopfer H, Mihatsch MJ: Plasma cell infiltrates in polyomavirus nephropathy. Transpl Int 2010, 23(4):397-406. Epub 2009/11/17.

24. Delbue S, Ferraresso M, Elia F, Belingheri M, Carloni C, Signorini L, et al: Investigation of polyomaviruses replication in pediatric patients with nephropathy receiving rituximab. J Med Virol 2012, 84(9):1464-70. Epub 2012/07/25.

25. Binet I, Nickeleit $V$, Hirsch HH, Prince O, Dalquen P, Gudat F, et al: Polyomavirus disease under new immunosuppressive drugs: a cause of renal graft dysfunction and graft loss. Transplantation 1999, 67(6):918-22. Epub 1999/04/13.

26. Nickeleit $V$, Hirsch $H H$, Binet IF, Gudat F, Prince $O$, Dalquen $P$, et al: Polyomavirus infection of renal allograft recipients: from latent infection to manifest disease. J Am Soc Nephrol 1999, 10(5):1080-9. Epub 1999/05/08.

27. Schold JD, Rehman S, Kayle LK, Magliocca J, Srinivas TR, Meier-Kriesche HU: Treatment for BK virus: incidence, risk factors and outcomes for kidney transplant recipients in the United States. Transpl Int 2009, 22(6):626-34. Epub 2009/02/12.

28. Toyoda M, Puliyanda DP, Amet N, Baden L, Cam V, Radha R, et al: Coinfection of polyomavirus-BK and cytomegalovirus in renal transplant recipients. Transplantation 2005, 80(2):198-205. Epub 2005/07/26.

29. Howell DN, Smith SR, Butterly DW, Klassen PS, Krigman HR, Burchette JL Jr, et al: Diagnosis and management of BK polyomavirus interstitial nephritis in renal transplant recipients. Transplantation 1999, 68(9):1279-88. Epub 1999/11/26.

30. Bielorai B, Shulman LM, Rechavi G, Toren A: CMV reactivation induced BK virus-associated late onset hemorrhagic cystitis after peripheral blood stem cell transplantation. Bone Marrow Transplant 2001, 28(6):613-4. Epub 2001/10/19.

31. Erard V, Storer B, Corey L, Nollkamper J, Huang ML, Limaye A, et al: BK virus infection in hematopoietic stem cell transplant recipients: frequency, risk factors, and association with postengraftment hemorrhagic cystitis. Clin Infect Dis 2004, 39(12):1861-5. Epub 2004/12/04. 
32. Liacini A, Seamone ME, Muruve DA, Tibbles LA: Anti-BK virus mechanisms of sirolimus and leflunomide alone and in combination: toward a new therapy for BK virus infection. Transplantation 2010, 90(12):1450-7. Epub 2010/11/17.

33. Egli A, Kohli S, Dickenmann M, Hirsch HH: Inhibition of polyomavirus BKspecific T-Cell responses by immunosuppressive drugs. Transplantation 2009, 88(10):1161-8. Epub 2009/11/26.

34. Benavides CA, Pollard VB, Mauiyyedi S, Podder H, Knight R, Kahan BD: BK virus-associated nephropathy in sirolimus-treated renal transplant patients: incidence, course, and clinical outcomes. Transplantation 2007, 84(1):83-8. Epub 2007/07/14.

35. Wali RK, Drachenberg C, Hirsch HH, Papadimitriou J, Nahar A, Mohanlal V, et al: BK virus-associated nephropathy in renal allograft recipients: rescue therapy by sirolimus-based immunosuppression. Transplantation 2004, 78(7):1069-73. Epub 2004/10/14.

36. Sanchez Fructuoso Al, Calvo N, Perez-Flores I, Valero R, Rodriguez-Sanchez B, Garcia Deviedma D, et al: Mammalian target of rapamycin signal inhibitors could play a role in the treatment of BK polyomavirus nephritis in renal allograft recipients. Transpl Infect Dis 2011, 13(6):584-91. Epub 2011/05/19.

37. Schwarz A, Linnenweber-Held S, Heim A, Brocker V, Rieck D, Framke T, et al: Factors Influencing Viral Clearing and Renal Function During Polyomavirus BK-Associated Nephropathy After Renal Transplantation. Transplantation 2012, 94(4):396-402. Epub 2012/07/28.

38. Gois PH, Rivelli GG, Pereira LM, Mazzali M: Sirolimus in renal transplantation: analysis of safety and efficacy in a nonprotocol conversion group. Transplant Proc 2012, 44(8):2348-51. Epub 2012/10/03.

doi:10.1186/1471-2369-14-207

Cite this article as: Jacobi et al.: BK viremia and polyomavirus nephropathy in 352 kidney transplants; risk factors and potential role of mTOR inhibition. BMC Nephrology 2013 14:207.

\section{Submit your next manuscript to BioMed Central and take full advantage of:}

- Convenient online submission

- Thorough peer review

- No space constraints or color figure charges

- Immediate publication on acceptance

- Inclusion in PubMed, CAS, Scopus and Google Scholar

- Research which is freely available for redistribution 